\title{
Demonstrating the sub-nanometer sensitivity of a pyramid WaveFrontSensor for active space telescopes
}

Runa Briguglio, Guido Agapito, Ciro Del Vecchio, Enrico Pinna, Marco Xompero, et al.

Runa Briguglio, Guido Agapito, Ciro Del Vecchio, Enrico Pinna, Marco Xompero, Carmelo Arcidiacono, Alessandro Terreri, Fernando Pedichini, "Demonstrating the sub-nanometer sensitivity of a pyramid WaveFrontSensor for active space telescopes," Proc. SPIE 11852, International Conference on Space Optics - ICSO 2020, 1185251 (11 June 2021); doi: $10.1117 / 12.2599825$

SPIE Event: International Conference on Space Optics - ICSO 2021, 2021, Online Only 


\section{International Conference on Space Optics-ICSO 2020}

Virtual Conference

30 March-2 April 2021

Edited by Bruno Cugny, Zoran Sodnik, and Nikos Karafolas
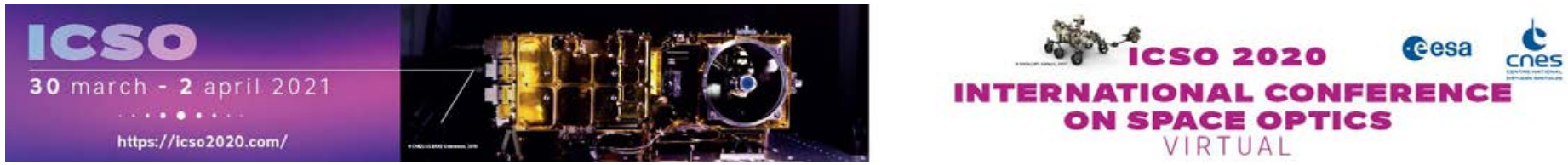

\section{Demonstrating the sub-nanometer sensitivity of a pyramid WaveFrontSensor for active space telescopes}

\section{Cesa isoporecestings denes}




\title{
Demonstrating the sub-nanometer sensitivity of a pyramid WaveFrontSensor for active space telescopes.
}

\author{
Runa Briguglio ${ }^{a}$, Guido Agapito ${ }^{\mathrm{a}}$, Ciro Del Vecchio ${ }^{\mathrm{a}}$, Enrico Pinna ${ }^{\mathrm{a}}$, Marco Xompero ${ }^{\mathrm{a}}$, \\ Carmelo Arcidiacono ${ }^{\mathrm{b}}$, Alessandro Terreric ${ }^{\mathrm{c}}$, and Fernando Pedichinic ${ }^{\mathrm{c}}$ \\ ${ }^{a}$ INAF Osservatorio Astrofisico Arcetri L. E. Fermi 5, 50125 Firenze Italy \\ bINAF Osservatorio Astronomico Padova, Vicolo dell'Osservatorio, 5, 35122 Padova Italy \\ 'INAF Osservatorio Astronomico Roma, Via Frascati 33, 00078 Monte Porzio Catone (Roma) \\ Italy
}

\begin{abstract}
In order to deliver high contrast, high resolution images, a keypoint in future space telescopes is to implement a correction chain, composed by a WaveFront Sensor (WFS) and an active/deformable mirror (DM) running in closed loop. While the current baseline for WFS is mostly focused on PSF sensing, no or little attention has been paid to a crucial element: after an initial startup phase, a space telescope will be operating in quasi diffraction-limited regime, so that a WFS shall be able to stably measure nanometer-level aberrations. In this scenario, the aberration signal in the PSF is overwhelmed by the bright core and to get rid of the photon noise a long time integration is required, which is converted into a more stringent stability requirement for the DM. We propose the implementation of the pyramid WFS (PWFS), which has been already demonstrated on-sky for high contrast at large ground based observatories. In this paper we present numerical simulations of a PWFS controlling an active primary in space. We will show the signal measured by the pyramid when a offset is applied on the mirror and the closed loop performances. The results indicate that the PWFS is able to detect nanometer-level low spatial scale aberrations and drive the active mirror with an optical stability consistent with typical requirements.
\end{abstract}

Keywords: Space telescopes, pyramid wavefront sensor, correction chain, high contrast, space active optics

\section{INTRODUCTION}

In the last decade the interest of the astronomical community focused on a new concept of large space telescope, intended for high contrast imaging in UV-optical-IR bands. The top-level science objectives descend from the vast heritage of the HST: for instance, the origin of cosmic structures, the detection and characterization of exoplanets and the search for signatures of life in exo-atmospheres. Such an interest resulted in several conceptual designs and notional architectures: HDST (High Definition Space Telescope), ATLAST (Advanced Technology Large Aperture Space Telescope), LUVOIR (Large UV-Optical-IR surveyor). All of these designs are based on a segmented primary, 8 to $15 \mathrm{~m}$ in diameter, to be deployed in space; operational wavelength coverage from UV to K-band; diffraction limited in V-band, WF (Wavefront) stability better than $1 \mathrm{~nm}$ per control step, pointing accuracy better than 1 milliarcsec. Compared with the traditional boundaries of space missions, such requirements pose unprecedented technological challenges, together with high mission risk and cost. An overview of these concepts may be found in the references (see for instance 1,2,3,4,5,6). A key point, common to many groups, is that the active control of the optical surfaces may help reducing top level tolerances, while improving the performances and simplifying global-wide the architecture. Such a concept, also known as active/adaptive optics, is routinely used in ground based large telescopes: a WF sensor reads the WF deformations due to telescope out-of-shape or atmospheric turbulence; a control loop converts such a signal into a compensation command, applied by a WF corrector element (a secondary or instrument/post-focal deformable mirror). Many

Further author information:

Runa Briguglio: E-mail: runa.briguglio@inaf.it, Telephone: +39 0552752200 
recipes were developed for the WF corrector (see for instance 7,8,9,10,12,13), including diverse actuators, mirror technology, correction strategy. On the other hand, WFS and control loop strategies are mostly focused on PSF sensing, since it may be easily implemented on the scientific camera or on a dedicated detector with no additional devices.

The PSF sensing has some drawbacks because in order to get an optimal result the most assessed methods require the injection of peculiar well known aberrations (i.e.defocus ) in order to remove the degeneration of the solutions in the wavefront analysis. Examples can be found in literature $(14,15)$, while different kind of image analysis are being conducted worldwide (16) and within our team, with the aim to skip the aberration injection and use direct sharp science fresh images from the telescope focal plane, maximizing the scientific efficiency during the observation ( 17).

We focus here on the case of a large diameter telescope, optimized for $\mathrm{V}$ band observations and operating (in space) at the quasi diffraction limit regime. In such conditions the WFS shall be able to detect with a robust SNR deviations of the WFE at the level of a few nanometers. Moreover, when the primary has $n$ segments on a diameter $d$, the spatial scales of interest are in the range of $1 \sim n \lambda /$ d. Especially when using coronagraph for the detection of heart-like exoplanets, whose contrast is around $10^{-10}$, also WF aberrations less than $10 \mathrm{~nm}$ may worsen the target detection; in fact this represents differential luminosity of the PSF comparable to the luminosity of the planet itself. Here in case of aberration mitigation by direct focal plane PSF analysis, the signal to be detected is therefore extremely faint and very close to the overwhelming bright central core of the PSF itself. Simulations of Mag-R 12 stars on a 8 meter space telescope shows that several hours of integration are needed to detect such a low signal, not taking into account the issues related to the PSF core saturation on the detector.

We propose a non-mainstream approach and to adopt a pupil-plane WFS: in particular the pyramid WFS (PWFS) for its intrinsic superior sensitivity to low spatial scale aberrations and phase steps.

\section{THE PYRAMID WAVEFRONT SENSOR}

The pyramid WFS implements the knife edge test and is basically composed by a pyramid prism, with its tip placed at the telescope focal plane. The light beam is split by the four faces, then a relay lens produces four pupil images on a detector. Any deviation from the diffraction limited PSF on the pyramid tip results in an intensity unbalance amongst the 4 images, so that it is possible to evaluate the WF slope by comparing the 4 images. The algorithm for the slope computation is described in 18. The pyramid WFS, which has been originally proposed and developed in Italy at INAF (Italian Institute for Astrophysics) has been proven to be a very effective solution for high resolution in many Adaptive Optics (AO) systems $(20,21,22,23,24)$ so that it has been selected for several future systems (refer to 26 27, 28, 29), including those for the Extremely Large Telescopes (GMT, ELT). The PWFS presents in facts two main elements. As first, it exploits the full telescope resolution to sense the WF: the larger the diameter, producing a sharper PSF, the better the WF sensitivity, which in turn is spatial-scale dependent (more sensitive at the lowest spatial scale). As a second point, the PWFS is intrinsically sensitive to phase steps, i.e vertical offsets in the WF map; so that it allows measuring and recovering the differential piston (and differential alignment in general) amongst the mirror segments after deployment and tracking them in close loop during the observations. This behaviour has been demonstrated both in simulation and in laboratory testing (31).

Along with the development of PWFS systems, simulation codes were setup to help designing and optimizing the instruments and to investigate innovative control strategies. One of the available AO simulator including the PWFS is PASSATA (30) : it has been originally prepared for the Large Binocular Telescope (LBT) First Light AO systems and has been proven to be a accurate tool to predict the AO loop performances (21); it has also been expanded to allow simulating future instruments such as the those for the ELTs as shown in 26, 27, 28.

PASSATA models the operation of an AO system from the propagation of a wavefront through the atmosphere up to the formation of the image on the detector of the WFS. It comprises the WF correction thanks to DMs, the real time computer (RTC) and the auxiliary control loops. The main outputs are the PSF on the science camera and the residual WF map on the pupil. The module integrates also ancillary algorithms to calibrate the AO system interaction matrix (linking mirror commands and WFS signals), to measure the slope null pattern, 
i.e. the signal due to diffraction registered with zero WFE, and to measure and optimize the optical modal gain. PASSATA has been recently integrated with a module to simulate a large aperture, segmented, active primary mirror and investigate the feasibility of a PWFS for space telescope. Such activity is performed within the SPLATT project.

\section{THE SPLATT SIMULATION}

The SPLATT project is a work funded by INAF aiming at the technological transfer in the field of astronomical adaptive optics. A major goal of the work is to demonstrate the usability of the PWFS for active correction chain in space. The objective, at the current stage, is pursued by means of numerical simulations, also leveraging on the strong expertise matured at INAF in end-to-end simulation of deformable mirrors, WFS and correction loops. The main questions to be addressed in this work are:

1. what is the sensitivity of a PWFS to low-mid spatial scales aberrations, those affecting mostly the coronagraphic contrast, in quasi diffraction limited regime?

2. how is noise propagated in an AO chain with PWFS and what is its impact on the stability of the WFE?

3. what is the sensitivity of a PWFS to the differential piston amongst segments and what is its stability after convergence?

With these questions in mind, we developed a numerical code to create a segmented, deformable primary mirror and integrated it into PASSATA. The primary mirror is a LATT-way (see for instance 12, 13) active thisshell system controlled by voice coil actuators. The concept shares the same technology already implemented and extensively verified for the adaptive secondaries of the Large Binocular Telescope (LBT) and Very Large Telescope (VLT), so that the simulation toolkit is robust and mature. The numerical code runs in two main steps: the individual deformable segment is simulated by means of a Finite Elements (FE) model, including the glass thin shell, the actuators, the electro-mechanical calibration of the system; the primary mirror is then assembled by replicating the segments (i.e. the FE models) according to a given geometry. These steps are described in the following.

\subsection{Creation of the primary mirror assembly}

The individual mirror segment is created with a FE model, including both the thin glass plate and the actuators. The model is able to create the mirror deformation when an actuator command is applied. Each FE realization represents a peculiar DM, considered here as an individual mirror segment. Each segment is independent, with respect to the others under the control point of view: in facts actuators and positioners act on that specific segment only. The lack of cross-talk amongst segments allows us to replicate the FE model of the single segment and populate the entire primary mirror system. This task is accomplished in three stages.

As first, the geometry of the primary is drawn, according to the following procedure:

1. the segment arrangement is defined: since the segments are hexagonal in shape, we considered an hexagonal grid, which is also a suitable arrangement to minimize the impact of aperture filling factor and obstructions in the final PSF;

2. given the number of segments on the M1 diameter, the total number of segments is computed together with the segments coordinates in the grid; at this stage, a mechanical gap is considered amongst segments;

3. a segment mask is placed at each segment coordinate to populate the primary; the mirror aperture is then defined as a mosaic of the segment hexagonal masks;

4. the primary mirror aperture is then cut to the largest circular shape, to mitigate the impact of the hexagonal grid on the PSF. 
As a second step we prepare the control matrices for the primary mirror. They are basically block diagonal matrices, obtained by arranging the individual contributions from the segments. Here we recall that we are considering voice coil deformable mirrors, whose actuators work in closed loop fed by co-located position capacitive sensors. Such systems are controlled by means of the so-called feed-forward matrix, which represents the system stiffness. Operatively, it is defined as the coefficient $k$ (in matrix form) of the Hook law between the force $F$ applied to actuators and the displacement $d$ read by the capacitive sensors:

$$
F=-k d \text {. }
$$

Such a relation is evaluated by the FE model. The $k$ matrix is not diagonal: since the actuators are in close loop, when a single one is pushed, the neighbouring will apply a counteracting force to keep their starting position. With the singular values decomposition of the $k$ matrix we obtain the position and force eigenvectors, $V$ and $U$. The shapes in the $V$ matrix, in particular, represent the eigenmodes of the DM, considered as an electromechanical system (practically, they resemble the thin plate modes). The $V$ matrix is a very convenient control space, since the largest DM deformation comes typically from low order modes, which may be measured with very large SNR and are controlled with a low force budget. In the end, the primary mirror control matrix is a block-diagonal, (Nact x Nsegm) x (Nact x Nsegm) containing the actuator commands to produce each of the individual segment eigenmode.

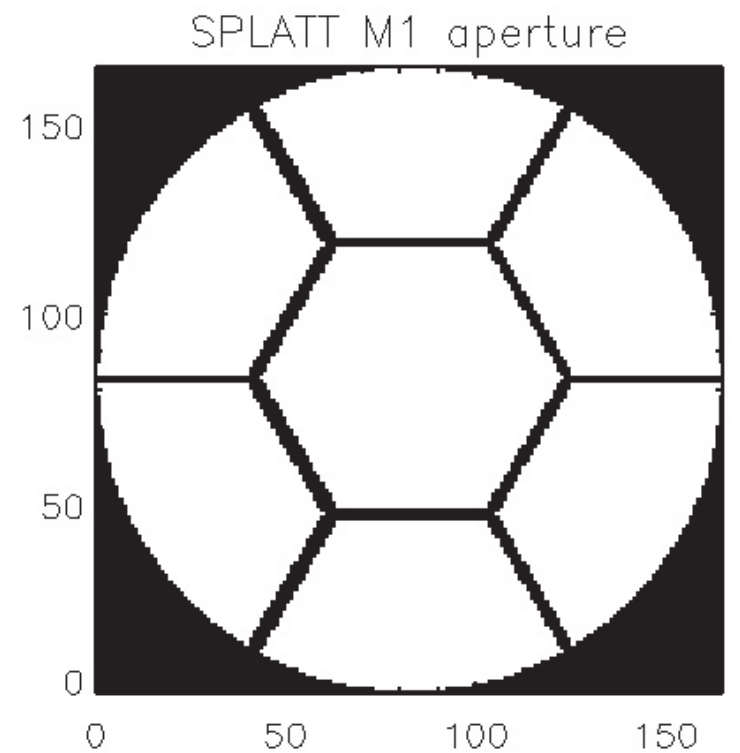

Figure 1. Pupil mask, or the geometry of the segmented primary mirror. A circular mask is added to the hexagonal arrangement of the segments to reduce the impact of diffraction.

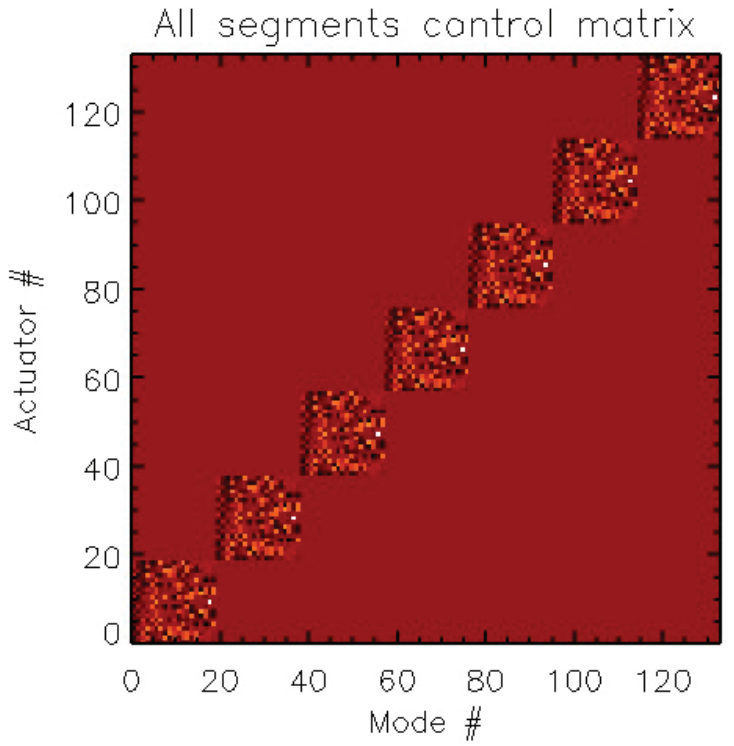

Figure 2. Block-diagonal matrix of the all-segments mirror modes.

As a third steps we created the Influence Functions (IF) matrices; an IF matrix is a Nact x Npixel matrix representing the primary mirror surface maps after a given excitation (here Npixel is the number of pixels within the aperture mask) . We identified four cases, as in the following.

- actuators IF: represent the mirror surface when a single actuator is poked, while the neighbouring ones apply a counter-force to keep the zero-displacement.

- mirror modes IF: represent the mirror surface when a mirror mode is applied; a mirror mode is a vector in the $V$ matrix. 
- segment Zernikes IF: represent the expected mirror surface when a single segment is out-of-place, i.e. tilted wrt the others or at a differential piston.

- global Zernikes IF: Zernike surfaces, drawn on the primary mirror aperture mask, representing the alignment aberrations (tilt to spherical aberrations).

Actuators and mirror modes IF are produced with the FE model of the segment, so that they shall be specifically produced for any segment size, geometry and actuators arrangement. Zernike shapes, on the contrary, are drawn on-the-fly with library routines.

The procedure depicted above is generalized and may be run to produce any primary mirror model, starting from M1 diameter, size and arrangement of the segments and FE model of the segment.

As a last step, the so-called disturbance maps are created, representing the pupil surface maps to be corrected by
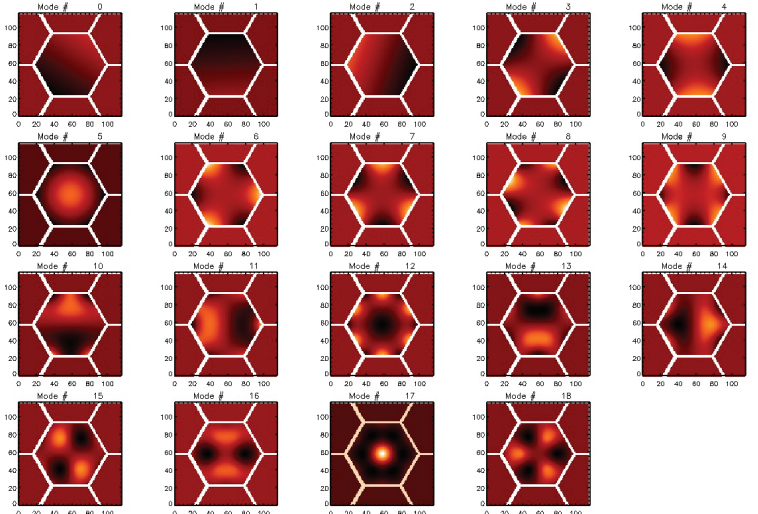

Figure 3. Surface map of the mirror modes of segment \#1.
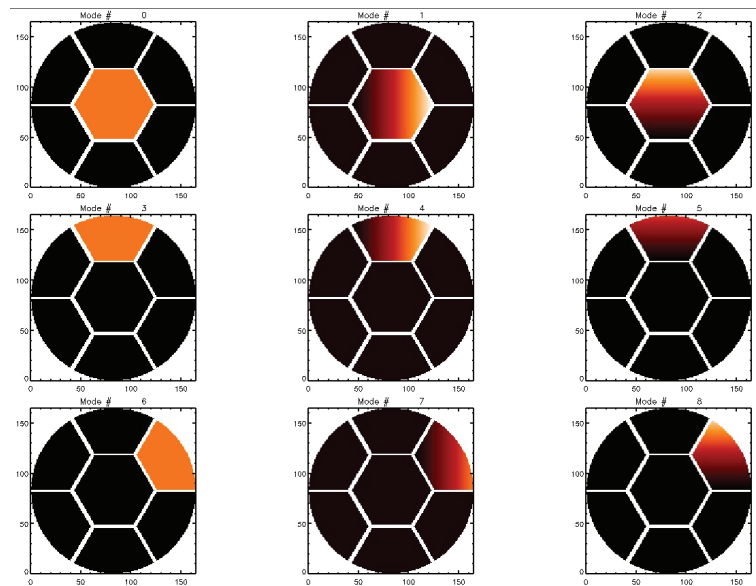

Figure 4. Surface map of the piston-tip-tilt modes of the segments \# 1, \#1, \#3.

the close loop. Basically, they are of two types: static offsets, which are a starting condition such as the initial misalignment of the segments or an initial actuators scramble; or time histories, i.e. a sequence of variable pupil surface maps, each associated with an individual loop step, so that their temporal frequency matches the loop one. In Tab. 1 we report the main parameters of the simulated primary mirror.

\begin{tabular}{|c|c|c|c|}
\hline M1 diameter & $2.04 \mathrm{~m}$ & Segment size & $1 \mathrm{~m}$ \\
\hline N. segm/diameter & 3 & N. segments & 7 \\
\hline N. actuators/segm. & 19 & Total act. count & 133 \\
\hline Inter-segm. gap & $0.1 \mathrm{~m}$ & & \\
\hline FEM: N. nodes & 7651 & Pixel pitch & $0.012 \mathrm{~m} / \mathrm{pix}$ \\
\hline N. pix/M1 diam. & 165 & N. pix/gap & 8 \\
\hline
\end{tabular}

Table 1. Overview of the parameters to create the simulated active primary mirror.

\subsection{Integration with PASSATA}

The active primary mirror is integrated with PASSATA by means of the mirror control matrices and the mask of the valid points within the aperture. Operatively, the integration is managed through a configuration file containing the identifier of the data to be used; then they are loaded into the system and the simulated primary mirror is created.

In the same way, the simulated PWFS is defined through a configuration file where the relevant WFS and loop parameters are indicated. At this stage, the simulated PWFS is started up in two steps. As first, the measurement mask is created: the camera is read-out (from a region corresponding to the entire pupil, illuminated with a 
flat wavefront) and the signal is processed into X, Y slopes; then a thresholding criterion allows rejecting the pixels with insufficient SNR. In parallel, the system records the sensor offset or slope null, which is the signal associated with a pure flat wavefront, and is mostly given by diffraction effects on the pyramid edges and roof. The subtraction of such a null signal is of paramount importance since we intend to work in a WF signal regime close to the diffraction limit.

The last step is the calibration of the active loop, consisting in the sequential excitation of the DM actuators, or DM degrees of freedom in general, and the registration of the corresponding PWFS slope signal. The result is the so-called interaction matrix, a npixel $\mathrm{x}$ nmodes table containing the PWFS slope associated with each of the DM modes. In Fig. 5 we show the slope signal associated with each of the DM degrees of freedom excited. In the case presented, the mode command amplitude is $20 \mathrm{~nm}$ RMS.
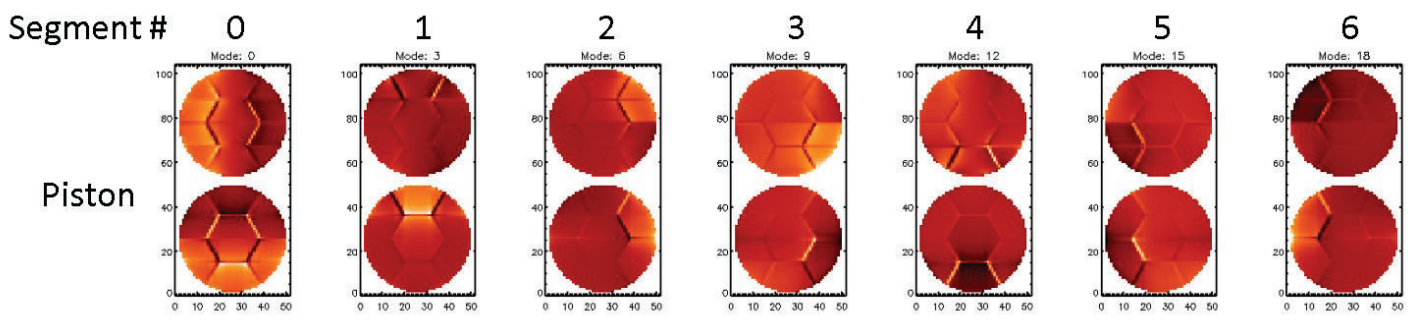

Tip
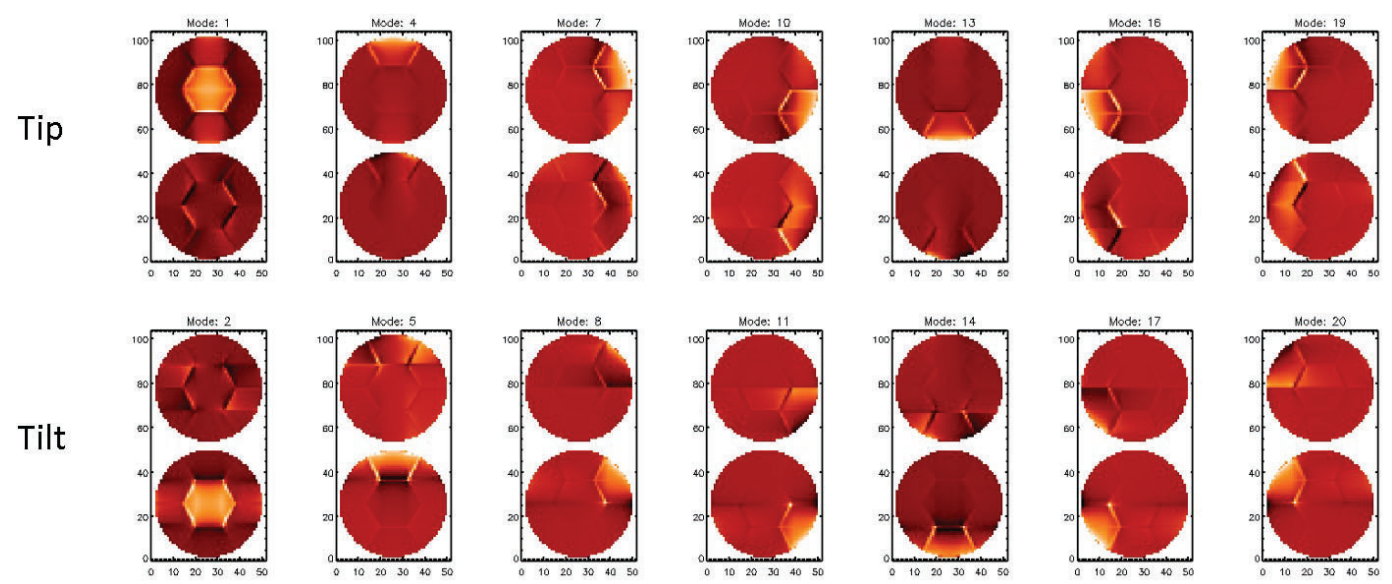

Figure 5. Optical interaction matrix of the system, or the signal recorded by the PWFS when a single degree of freedom of the DM is excited. In the present case we consider the segments alignment modes piston, tip and tilt. The upper and lower panels represent $\mathrm{X}$ and $\mathrm{Y}$ slope, respectively.

\subsection{The simulation parameters space}

As mentioned, we intend to investigate the PWFS sensitivity in the very low WFE regime. To this end, we considered a parameters space in the simulation as described in the following.

\section{DM offset}

We considered at this stage a DM offset given only by differential alignment amongst the segments, i.e. differential piston, tip, tilt. At a first step we exercise the system with a single segment piston-tilt error. Later we considered a random scramble of all the segments. In the end, the DM offset added in the loop is a $3 \mathrm{x} 7$ elements vector, representing the coefficients of the segments Zernike shapes. Regarding the offset amplitude, we normalized the offset map so that it was $100 \mathrm{~nm}$ RMS surface, a value that is sound when compared to $\mathrm{V}$ band and to the PWFS calibration amplitude (20 nm WF). The DM offset map is stored in a $165 \mathrm{x} 165$ pixel, with a pitch of $0.0125 \mathrm{~m} /$ pixel, to be compared with an inter-actuator distance of $20 \mathrm{~cm}$ and a segment gap of $5 \mathrm{~cm}$. 


\section{PWFS geometry}

The signal of an individual PWFS pupil image (out of the 4 ones) is created over a $48 \times 48$ pixel bounding box, with the image centers separated by 52 pixel. Such value are very robust to image with no aliasing errors the individual segments actuator influence functions, which are the smallest spatial scale to be measured. In this regard, the sensing of very low spatial scale modes doesn't require such a sampling and the simulation is therefore conservative. The PWFS is not modulated since modulation allows a larger capture range with a poorer sensitivity. Modulation may be however considered in an initial segment-recovery phase.

\section{AO loop}

The PWFS works at a wavelength of $750 \mathrm{~nm}$, with a badnwidth of $\pm 150 \mathrm{~nm}$. The guide star magnitude is userselectable and we run simulations in the magnitude range 8 to 12 (R-band). The loop timing is user-selectable as well and we considered as a baseline a time integration in the range $0.1 \mathrm{~s}-1 \mathrm{~s}$. The loop control is a pure integrator, with a 1 step delay (a longer delay, considering such a long time integration - compared to typical values for ground based AO- is not requested). The loop gain is a test parameter in the simulation and we considered values in the range $0.1-0.3$. The loop was found unstable for gains larger than 0.7. The gain is modal so that it is possible to tune it on a mode-by mode basis.

\section{Noise and throughput}

The simulation includes the photon noise and the camera noise. The former in particular is computed from the the guide star magnitude and considering a very conservative system throughput 0.32 (full telescope throughput and quantum efficiency of the camera). The detector noise is taken from a database (included in PASSATA) of noise parameters, measured in laboratory on several detectors used for WFS systems. In the present case we considered a value of $10 \mathrm{e}-$, including read-out noise and dark current.

\section{PERFORMANCES}

The simulation consists in a close loop to recover and track an initial DM offset, in the form of differential alignment (including differential piston) amongst the segments. After the initial PWFS calibration we completed several runs to explore the simulation parameters, in particular the guide star magnitude and the sensor time integration. For each run, we considered two indicators for the loop quality: the mean of the DM surface error RMS after convergence, and its dispersion. Referring to Fig.6, the former is the average of the values inside the red box, the latter is their standard deviation. This, in particular, is taken as an indication of the DM and loop stability. Each run consists of 1000 loop cycles, for a total simulated time in the range $300 \mathrm{~s}$ to $1000 \mathrm{~s}$, depending of the loop integration time. Such duration is consistent with a typical exposure time on a scientific target. The initial offset is shown (for that specific run) in the left panel in Fig.6. In Fig.7 we show the corresponding distribution histogram of the DM residues. The PWFS signal (X and Y slopes) are shown in Fig.8 for a few steps after starting the close loop. The initial offset signal is recovered and after convergence the slopes are given by the photon and detector noise.

In tab 2 we collected the convergence and stability values for a sample of runs: we exercised the system with a DM offset of $100 \mathrm{~nm}$ to $150 \mathrm{~nm}$ amplitude and closed the loop on a guide star with magnitude 8 to 12 .

The typical convergence value is below $3 \mathrm{~nm}$ RMS: comparing the results obtained at magnitude $12(0.1 \mathrm{~s}$ vs 0.3 time integration) we guess that the loop is possibly not photon noise-dominated. The loop results are also, as expected, not depending on the initial DM offset. A critical point is the piston control of the segments, in particular when the signal on the WFS (which has a periodic response vs lambda) approaches the lambda/2 threshold. We observed in facts that the loop diverges to a wrong piston identification when the DM offset is larger than $180 \mathrm{~nm} \mathrm{SE} \mathrm{(equal} \mathrm{to} 360 \mathrm{~nm} \mathrm{WF}$, with the threshold set naturally to $750 / 2 \mathrm{~nm} \mathrm{WF}$ ). For this reason we selected a DM offset amplitude lower than $150 \mathrm{~nm}$.RMS SE. Such point is not detrimental of the robustness of the simulation: we intend in facts to evaluate the stability of the PWFS in the low WFE regime, so that an initial offset of $100 \mathrm{~nm}$ to $150 \mathrm{~nm}$ is to be considered very conservative.

The WF stability at convergence is below $1 \mathrm{~nm}$ for all the simulated test cases. Such a result shall be compared with the typical stability requirement for the DM and optics in general. 
PWFS close loop
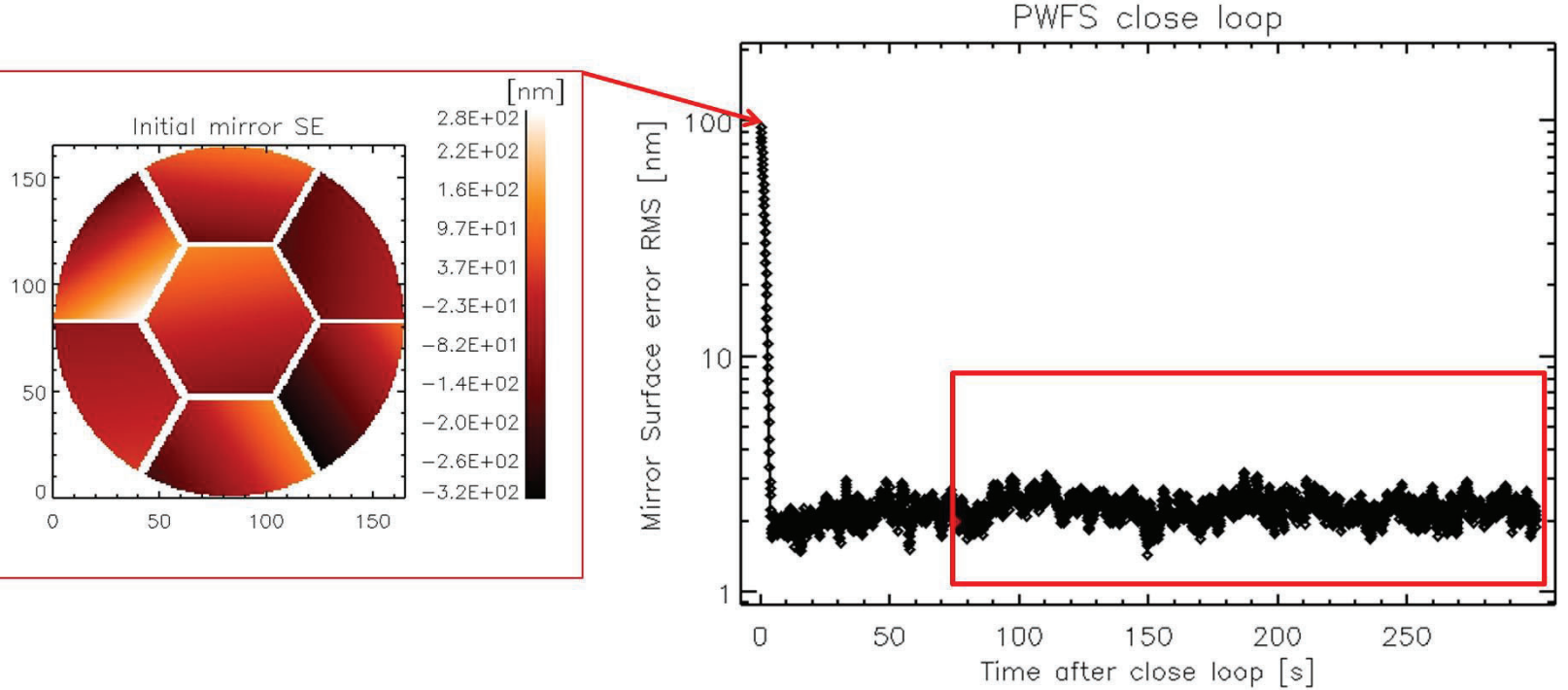

Figure 6. Mirror surface error, as a function of time during a simulated close loop run. The initial offset is $100 \mathrm{~nm}$ RMS, with a spatial distribution (for this peculiar run) as shown in the figure in the left panel: each segment is individually excited with a piston-tip/tilt offset. The red box indicates the sample for the evaluation of the loop quality, mean and dispersion of the surface error.

\begin{tabular}{|c|c|c|c|c|}
\hline DM SE offset $[\mathrm{nm}]$ & Mag & T.Exp $[\mathrm{s}]$ & SE mean $[\mathrm{nm}]$ & SE StDev $[\mathrm{nm}]$ \\
\hline 100 & 8 & 0.1 & 2.07 & 0.14 \\
\hline 100 & 10 & 0.1 & 2.29 & 0.26 \\
\hline 100 & 12 & 0.1 & 6.01 & 1.06 \\
\hline 100 & 8 & 0.3 & 2.06 & 0.13 \\
\hline 100 & 10 & 0.3 & 2.09 & 0.16 \\
\hline 100 & 12 & 0.3 & 2.85 & 0.44 \\
\hline 150 & 8 & 0.1 & 2.08 & 0.14 \\
\hline 150 & 10 & 0.1 & 2.29 & 0.27 \\
\hline
\end{tabular}

Table 2. Simulation parameters and loop quality indicators for a set of close loop tests.

\section{ROADMAP}

The SPLATT simulation is intended to provide a first, preliminary demonstration of the WF stability that can be achieved using a pupil-plane, pyramid WFS to drive an active space telescope. We considered a single, very specific test case, in terms of telescope geometry, actuators arrangement, WFS setup; in parallel, we explored a very limited volume in the parameters space. Such a test case is intended to answer a very specific question: what is the expected sensitivity of a PWFS in quasi diffraction limited regime. We showed a set of preliminary results, indicating that the PWFS is able to recover an initial surface error of $100 \mathrm{~nm}$ RMS, to reduce it below 10 $\mathrm{nm}$ ( $<3$ typ., depending on integration time and guide star magnitude) and to keep it at the convergence value with a scatter lower than $1 \mathrm{~nm}$. We consider such results as an indication to continue and extend the SPLATT simulation to further validate the concept. Two points in particular deserve attention. As first, no specific optimization of the system was considered here: for instance, the WFS mask was not tailored to maximize the SNR, i.e. to discard those pixel where the slope signal is too low. This could be a major improvement for the sensing of the differential piston. As a second point, the simulations triggered some questions and in particular:

- what is the best sensing strategy for the segments differential piston, also including the possibility to measure and recover an initially very large offset ( hundreds of microns)?

- is the result scalable to much larger systems, with much higher actuators count? how shall the PWFS 


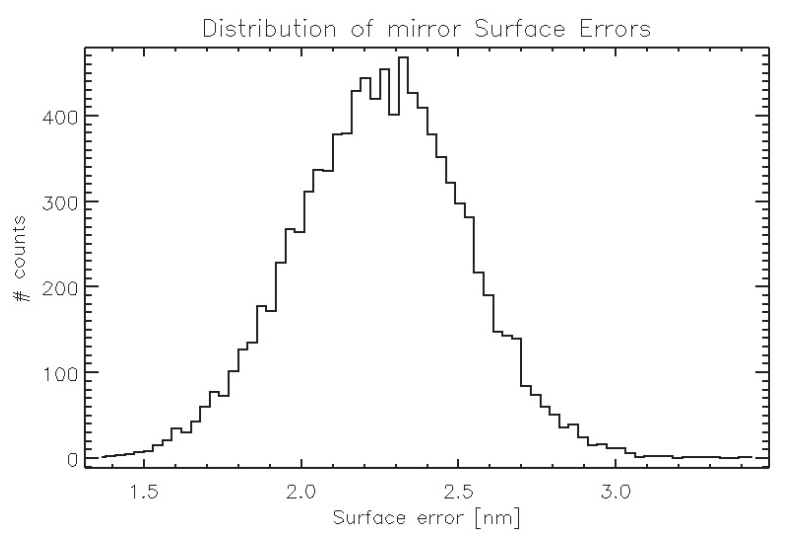

Figure 7. Distribution of the DM surface error after convergence.
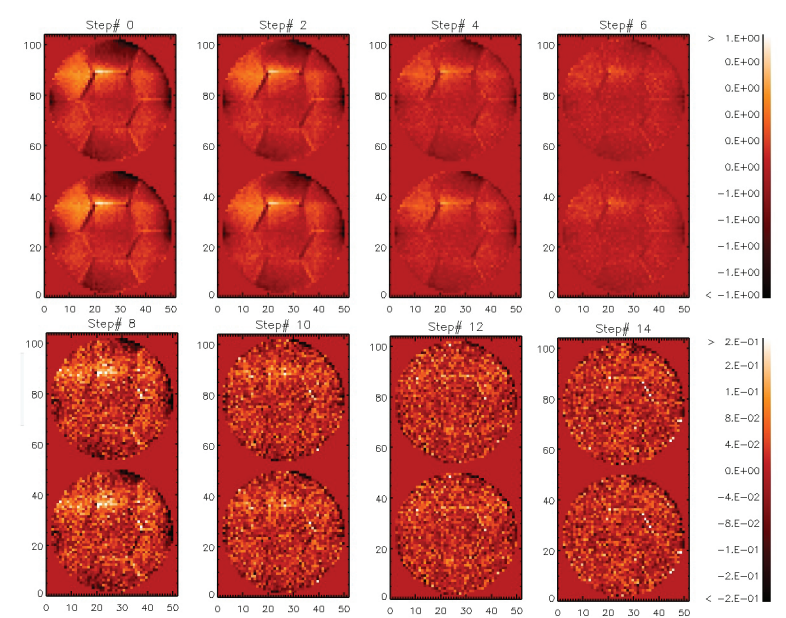

Figure 8. Slope signal, as measured by the PWFS during the initial stage of the close loop. The signal at step 0 is the one associated with the initial offset. When the loop recover the DM shape, the PWFS signal is basically due to the detector and photon noise.

adjusted accordingly, to match such more complex arrangements?

- will such results be maintained when an actuator scramble is added in the initial offset?

- how are these results compared with traditional PSF-sensing? may we identify a sensing and correction strategy that includes both approaches (pupil and PSF sensing) to maximize the performances?

The questions will be addressed more in detail within the activities of the SPLATT project at INAF. The project will possibly result in a robust roadmap for the development of WF sensing and active correction chains for space telescopes.

\section{REFERENCES}

[1] Feinberg, L., Cohen, L., Dean, B., \& al.: Space telescope design considerations, Optical Engineering 51, 2012.

[2] Bolcar, M., Balasubramanian, K., Crooke, J., \& al.: Technology gap assessment for a future large aperture ultraviolet-optical-infrared space telescope, JATIS 2, 2016.

[3] Feinberg, L., Bolcar, M., Knight, S., \& al.: Ultra stable segmented telescope sensing and control architecture, Proc of SPIE 10398, 2017

[4] Lightsey, P., Knight, S., Feinberg, L., \& al.: First order budgeting for LUVOIR mission, Proc of SPIE 10398, 2017

[5] Rioux, N., Thronson, H., Feinberg, L., Future large aperture UVOIR space observatory: reference designs, JATIS 2, 2016.

[6] Scott Acton, D., Scott Knight, J., Contos, A., \& al.: Wavefront sensing and controls for James Webb space telescope, Proc of SPIE 8442, 2012

[7] Hallibert, P., Zuccaro Marchi, A.: Developments in active optics for space instruments: an ESA perspectives, Proc of SPIE 9912, 2017.

[8] Rausch, P., Veerport, S. and Wittrock, U.: Unimorph deformable mirror for space telescope: design and manufacturing", Opt.Express 23 (2015), 19469-19477

[9] Kuiper, S., Doelman, N., et al.: Electromagnetic DM development at TNO, Proc of SPIE 9912 (2016) 
[10] Devaney, N., Goncharov, A. et al.: Development of a prototype Active Optics system for future space telescopes, Appl. Optics 57(22), 2018

[11] Steeves, J., Redding, D., Wallace, J., \& al.: Active mirrors for future space telescopes, Proc of SPIE 10706, 2018

[12] Briguglio, R., Del Vecchio, C., Lisi, F, \& al.: Toward large diffraction limited space telescopes with the LATT lightweight active primary, Proc of SPIE 10562, 2017

[13] Briguglio, Arcidiacono, C., Xompero, M, \& al.: The LATT way towards large active primaries for space telescopes, Proc of SPIE 9904, 2016.

[14] Roddier, C., Roddier, F.:Wave-front reconstruction from defocused images and the testing of groundbased optical telescopes, Journal of the Optical Society of America A Vol. 10, Issue 11, Nov 1993, DOI:10.1364/JOSAA.10.002277

[15] Mugnier, M. L., Blanc, A., Idier, J.: Phase Diversity: A Technique for Wave-Front Sensing and for Diffraction-Limited Imaging, Advances in Imaging and Electron Physics Volume 141, 2006, https://doi.org/10.1016/S1076-5670(05)41001-0

[16] Orban De Xivry, G., Louppe, G., Absil, O.,\& al.: Focal plane wavefront measurement with convolutional neural networks, MemSait, 1, 2019, https://doi.org/10.36116/WFS2019.24

[17] Terreri A., Mattioli M., Pedichini \& al.: Image Recognition for NCPA mitigation, MemSait, 1, 2019, https://doi.org/10.36116/WFS2019.23

[18] Ragazzoni R., 1996, JMOp, 43, 289.

[19] S. Esposito, A. Riccardi, Pyramid Wavefront Sensor behavior in partial correction Adaptive Optic systems A369 (2) L9-L12 (2001)

[20] Esposito, S., Riccardi, A., Fini, L. \& al.: First light AO (FLAO) system for LBT: final integration, acceptance test in Europe, and preliminary on-sky commissioning results, Proc. SPIE 7736, Adaptive Optics Systems II, 773609 (15 July 2010) https://doi.org/10.1117/12.858194

[21] Esposito, S., Riccardi, A., Pinna, E., \& al.: Large Binocular Telescope Adaptive Optics System: new achievements and perspectives in adaptive optics, Proc. SPIE 8149, Astronomical Adaptive Optics Systems and Applications IV, 814902 (16 September 2011); https://doi.org/10.1117/12.898641

[22] E. Pinna, S. Esposito, P. Hinz, G. Agapito, M. Bonaglia, A. Puglisi, M. Xompero, A. Riccardi, R. Briguglio, C. Arcidiacono, L. Carbonaro, L. Fini, M. Montoya, O. Durney, "SOUL: the Single conjugated adaptive Optics Upgrade for LBT," Proc. SPIE 9909, Adaptive Optics Systems V, 99093V (27 July 2016); https://doi.org/10.1117/12.2234444

[23] Close, L.M., Males, J. M., Kopon, D. K., \& al.: First closed-loop visible AO test results for the advanced adaptive secondary AO system for the Magellan Telescope: MagAO's performance and status, Proc. SPIE 8447, Adaptive Optics Systems III, 84470X (13 September 2012); https://doi.org/10.1117/12.926545

[24] Jovanovic, N. \& al.:2015 PASP 127890

[25] Males, J. M., Close, L., M., Guyon, O. \& al.: MagAO-X first light, Proc. SPIE 11448, Adaptive Optics Systems VII, 114484L (13 December 2020); https://doi.org/10.1117/12.2561682

[26] Pinna, E., Agapito, G., Quirós-Pacheco, F. \& al.: Design and numerical simulations of the GMT Natural Guide star WFS, Proc. SPIE 9148, Adaptive Optics Systems IV, 91482M (7 August 2014);

[27] Neichel 2016 10.1117/12.2231681,

[28] Neichel, B., Fusco, T., Sauvage, J-F.,\& al.: The adaptive optics modes for HARMONI: from Classical to Laser Assisted Tomographic AO, Proc. SPIE 9909, Adaptive Optics Systems V, 990909 (26 July 2016)

[29] Fitzsimmons, J., Agapito, G., Bonaglia, M. \& al.:GPI 2.0: design of the pyramid wave front sensor upgrade for GPI, Proc. SPIE 11448, Adaptive Optics Systems VII, 114486J (13 December 2020)

[30] Agapito, G., Puglisi, A., Esposito, S.:PASSATA: object oriented numerical simulation software for adaptive optics, Proc. SPIE 9909, Adaptive Optics Systems V, 99097E (27 July 2016)

[31] Bonaglia, M., Pinna, E., Puglisi, A., \& al.: First cophasing of a segmented mirror with a tunable filter and the pyramid wavefront sensor, Proc. SPIE 7739, Modern Technologies in Space- and Ground-based Telescopes and Instrumentation, 77392Y (22 July 2010); 